\title{
THE CIRCUMFERENCE OF A CONVEX POLYGON
}

\section{F. SPITZER AND H. WIDOM ${ }^{1}$}

In this note we combine a convexity theorem due to Cauchy with a combinatorial identity discovered by $\mathrm{M}$. Kac.

Cauchy's theorem [1] concerns the length $L$ of the circumference of a compact convex set $A$ in the plane. Let $D(\theta)$ denote the projection of $A$ on a line with direction $\theta, 0 \leqq \theta<\pi$, or, if $z=x+i y$

$$
D(\theta)=\max _{z \in A}(x \cos \theta+y \sin \theta)-\min _{z \in A}(x \cos \theta+y \sin \theta) .
$$

Then

$$
L=\int_{0}^{\pi} D(\theta) d \theta
$$

M. Kac, in [2], considered a vector $\mathrm{x}=\left(x_{1}, x_{2}, \cdots, x_{n}\right)$ with real components. For each permutation

$$
\sigma=\left\{\begin{array}{llll}
1 & 2 & \cdots & n \\
\sigma_{1} & \sigma_{2} & \cdots & \sigma_{n}
\end{array}\right\}
$$

he defined the vectors

$$
\mathbf{x}(\sigma)=\left(x_{\sigma_{1}}, x_{\sigma_{2}}, \cdots, x_{\sigma_{n}}\right),
$$

and their partial sums

$$
s_{0}(\sigma)=0, \quad s_{k}(\sigma)=x_{\sigma_{1}}+x_{\sigma_{2}}+\cdots+x_{\sigma_{k}}, \quad k=1,2, \cdots, n .
$$

His result may be stated in the form

$$
\sum_{\sigma}\left[\max _{0 \leqq k \leqq n} s_{k}(\sigma)-\min _{0 \leqq k \leqq n} s_{k}(\sigma)\right]=\sum_{\sigma} \sum_{k=1}^{n} \frac{1}{k}\left|s_{k}(\sigma)\right|
$$

where the $\sigma$-summation extends over the group of all permutations of $n$-objects.

We shall consider a vector $z=\left(z_{1}, z_{2}, \cdots, z_{n}\right)$ with complex components. As above we let

$$
\begin{aligned}
z(\sigma) & =\left(z_{\sigma_{1}}, z_{\sigma_{2}}, \cdots, z_{\sigma_{n}}\right), \\
s_{0}(\sigma) & =0, \quad s_{k}(\sigma)=z_{\sigma_{1}}+z_{\sigma_{2}}+\cdots+z_{\sigma_{k}}, \quad k=1,2, \cdots, n .
\end{aligned}
$$

Received by the editors May 14, 1960 and, in revised form, July 8, 1960.

${ }^{1}$ Research supported by the Office of Naval Research at the University of Minnesota. 
We define the set $A(\sigma)$ as the smallest convex set (polygon) containing all the points $s_{0}(\sigma), s_{1}(\sigma), \cdots, s_{n}(\sigma)$, and $L(\sigma)$ as the length of the circumference of $A(\sigma)$. We shall obtain the following generalization of equation (3).

\section{THEOREM 1.}

$$
\sum_{\sigma} L(\sigma)=2 \sum_{\sigma} \sum_{k=1}^{n} \frac{1}{k}\left|s_{k}(\sigma)\right| .
$$

Here the summation again extends over all permutations, and (4) is equivalent to (3) when all $z_{k}$ are real. When the $z_{k}$ are not real we write

$$
\begin{array}{rlr}
z_{k} & =x_{k}+i y_{k}, \quad z_{\sigma_{k}}=x_{\sigma_{k}}+i y_{\sigma_{k}}, & \\
t_{k}(\theta) & =x_{k} \cos \theta+y_{k} \sin \theta, & 0 \leqq \theta<\pi, \\
u_{0}(\theta, \sigma) & =0, \quad u_{k}(\theta, \sigma)=t_{\sigma_{1}}(\theta)+\cdots+t_{\sigma_{k}}(\theta), & k=1,2, \cdots, n .
\end{array}
$$

Let $D(\theta, \sigma)$ be the projection of $A(\sigma)$ on a line with direction $\theta$. Since $A(\sigma)$ is the convex hull of its extreme points we have from (1)

$$
D(\theta, \sigma)=\max _{0 \leqq x \leqq n} u_{k}(\theta, \sigma)-\min _{0 \leqq k \leqq n} u_{k}(\theta, \sigma) .
$$

By equation (2)

$$
L(\sigma)=\int_{0}^{\pi}\left[\max _{0 \leqq k \leqq n} u_{k}(\theta, \sigma)-\min _{0 \leqq k \leqq n} u_{k}(\theta, \sigma)\right] d \theta .
$$

By equation (3)

$$
\sum_{0} L(\sigma)=\sum_{\sigma} \sum_{k=1}^{n} \frac{1}{k} \int_{0}^{\pi}\left|u_{k}(\theta, \sigma)\right| d \theta .
$$

But

$$
\int_{0}^{\pi}\left|u_{k}(\theta, \sigma)\right| d \theta=2\left[\left\{\sum_{i=1}^{k} x_{\sigma_{i}}\right\}^{2}+\left\{\sum_{i=1}^{k} y_{\sigma_{i}}\right\}^{2}\right]^{1 / 2}=\left|s_{k}(\sigma)\right| .
$$

Hence (4) is proved.

As an application we derive a result of probabilistic interest. Let $Z_{1}, Z_{2}, \ldots$ denote a sequence of identically distributed independent complex valued random variables. Thus the distribution of each $Z_{k}$ is the same planar Lebesgue-Stieltjes measure and their joint distributions are given by the obvious product measure. We define their partial sums as the random variables 


$$
S_{0}=0, \quad S_{n}=Z_{1}+Z_{2}+\cdots+Z_{n}, \quad n \geqq 1 .
$$

Finally, let $L_{n}$ be defined as the length of the circumference of the smallest convex set containing $S_{0}, S_{1}, \cdots, S_{n}$. If " $E$ " denotes expectation with respect to the product measure, we have

THEOREM 2.

$$
E\left(L_{n}\right)=2 \sum_{k=1}^{n} \frac{1}{k} E\left|S_{k}\right| .
$$

The proof is based on two observations. First, $L_{n}$ is a continuous function of $S_{1}, S_{2}, \cdots, S_{n}$, so that it is a random variable. Secondly, if we define the random variables $Z_{\sigma_{k}}, S_{k}(\sigma), L_{n}(\sigma)$ as was done in the deterministic case, it follows from the invariance of the product measure under permutations $\sigma$ that the expectations $E\left|S_{n}(\sigma)\right|$ and $E\left(L_{n}(\sigma)\right)$ are independent of $\sigma$. This proves the theorem and also shows that either both sides in (5) are finite or neither. Of course they are finite if and only if $E\left|Z_{1}\right|<\infty$.

Finally, we consider two situations where the asymptotic behavior of $E\left(L_{n}\right)$ is of some interest.

(a) Let $Z_{1}, Z_{2}, \cdots$ be identically distributed and independent with

$$
\begin{array}{rlrl}
Z_{k} & =X_{k}+i Y_{k}, & & E\left(X_{k}\right)=E\left(Y_{k}\right)=0, \\
E\left(X_{k}^{2}\right)=a^{2}<\infty, & E\left(Y_{k}^{2}\right)=b^{2}<\infty, \quad E\left(X_{k} Y_{k}\right)=\rho a b .
\end{array}
$$

Then $n^{-1 / 2}\left(Z_{1}+\cdots+Z_{n}\right)$ has a bivariate normal limiting distribution and its absolute value may be shown to be uniformly integrable in $n$, so that

$$
\begin{aligned}
\lim _{n \rightarrow \infty} n^{-1 / 2} E\left|Z_{1}+\cdots+Z_{n}\right| \\
=(2 \pi)^{-1 / 2} \int_{0}^{\pi}\left[a^{2} \sin ^{2} \theta+b^{2} \cos ^{2} \theta+2 a b \rho \sin \theta \cos \theta\right]^{1 / 2} d \theta=c .
\end{aligned}
$$

It follows from Theorem 2 that

$$
\lim _{n \rightarrow \infty} n^{-1 / 2} E\left(L_{n}\right)=4 c .
$$

(b) Here we let $X_{1}, X_{2}, \cdots$ be a sequence of identically distributed independent random variables with

$$
E\left(X_{k}\right)=\mu, \quad E\left[\left(X_{k}-\mu\right)^{2}\right]=\sigma^{2}<\infty .
$$

We define the complex valued random variables 


$$
Z_{k}=X_{k}+i
$$

and their partial sums

$$
S_{0}=0, \quad S_{n}=X_{1}+\cdots+X_{n}+n i, \quad n=1,2, \cdots .
$$

The law of large numbers asserts that $n^{-1} S_{n} \rightarrow \mu+i$ with probability one. Geometrically this means that the polygonal path consisting of the points $S_{0}, S_{1}, \ldots$ does not deviate too far from the straight line through 0 and $\mu+i$. We shall denote by $L_{n}$ the circumference of the smallest convex set containing the points $S_{0}, S_{1}, \cdots, S_{n}$, and quite naturally, study the excess of $L_{n}$ over its smallest possible value, which is $2 n\left(1+\mu^{2}\right)^{1 / 2}$.

Theorem 2 yields

$$
\begin{aligned}
\frac{1}{2} E\left(L_{n}\right) & -n\left(1+\mu^{2}\right)^{1 / 2} \\
= & \sum_{k=1}^{n} E\left[\left\{\left(\frac{X_{1}+X_{2}+\cdots+X_{k}}{k}\right)^{2}+1\right\}^{1 / 2}-\left(\mu^{2}+1\right)^{1 / 2}\right] .
\end{aligned}
$$

Using the second order Taylor expansion of $\left(t^{2}+1\right)^{1 / 2}$ about $t=\mu$, it is quite simple to show that, as $k \rightarrow \infty$,

$$
\begin{aligned}
& E\left[\left\{\left(\frac{X_{1}+\cdots+X_{k}}{k}\right)^{2}+1\right\}^{1 / 2}-\left(\mu^{2}+1\right)^{1 / 2}\right] \\
& \quad \sim \frac{1}{2}\left(1+\mu^{2}\right)^{-3 / 2} E\left[\left(\frac{X_{1}+\cdots+X_{k}-\mu}{k}\right)^{2}\right]=\frac{1}{2}\left(1+\mu^{2}\right)^{-3 / 2} \frac{\sigma^{2}}{k} .
\end{aligned}
$$

It follows that

$$
\lim _{n \rightarrow \infty}(\log n)^{-1}\left[E\left(L_{n}\right)-2 n\left(1+\mu^{2}\right)^{1 / 2}\right]=\sigma^{2}\left(1+\mu^{2}\right)^{-3 / 2} .
$$

\section{REFERENCES}

1. H. G. Eggleston, Convexity, Cambridge, University Press, 1958.

2. M. Kac, Toeplitz matrices, translation kernels, and a related problem in probability theory, Duke Math. J. vol. 21 (1954) pp. 501-509.

UNIVERSITY OF MiNNESOTA AND

CORNELl UNIVERSITY 